\section{AB0729 ASSOCIATIONS BETWEEN CARDIAC CONDUCTION AND DISEASE CHARACTERISTICS IN AXIAL SPONDYLOARTHRITIS}

S. S. Zhao ${ }^{1,2}$, N. L. Harrison ${ }^{2}$, J. Ang ${ }^{2}$, N. Goodson ${ }^{1,2} .{ }^{1}$ University of Liverpool, Liverpool, United Kingdom; ${ }^{2}$ Aintree University Hospital, Liverpool, United Kingdom

Background: Cardiac conduction defects are well-documented in axial spondyloarthritis. However, historical literature (many from an era when axSpA was less well managed compared to modern day) include patients with advanced disease that may explain their high prevalence. Many recent studies reply on administrative codes that may under-report conduction defects. Thorough examination of ECG measurements and axSpA characteristics are scarce.

Objectives: To describe a range of cardiac conduction measurements in axSpA and their association with disease characteristics.

Methods: We conducted a single-centre cross-sectional study of consecutive patients meeting the ASAS axial SpA criteria in Liverpool, UK. Patients were excluded if they had a known/symptomatic conduction defect. Disease assessment included BASDAI, spinal pain, BASFI, CRP, ESR, HLA-B27, BMI, the presence of extra-articular manifestations (uveitis, psoriasis, IBD) and use of NSAIDs and TNFi. Each patient underwent a 12-lead ECG (GE healthcare; MAC2000) to obtain: PR (atrio-ventricular conduction), QRS (ventricular depolarization) and QTc (ventricular de- and repolarization) intervals in milliseconds (ms). QTc was corrected for heart rate using Bazett's formula. Prolonged PR interval was defined as $>200 \mathrm{~ms}$, prolonged QRS as $>100 \mathrm{~ms}$ and prolonged QTc as $>440 \mathrm{~ms}$ in men and $>460 \mathrm{~ms}$ in women. QT dispersion has been shown to predict a range of cardiac outcomes; we measured this as the difference between the longest and shortest QT in two consecutive cardiac cycles. Associations between patient characteristics and ECG measurements were assessed using univariable linear or logistic regression. Bonferroni correction was applied for multiple comparisons.

Results: 163 patients underwent ECG testing: mean age 52 (SD14) years, mean symptom duration 10 years (SD 9.6), 79\% male and 74\% HLA-B27 positive (among 78 tested). 1 patient had Wolf-Parkinson-White (accessory pathway). Summary of the 4 measurements are shown in Table 1. None of these 4 ECG measures were associated with age, symptom duration, gender, BMI, disease severity (BASDAI, spinal pain, BASFI and log transformed CRP/ESR), HLA-B27, EAMs or NSAIDs/TNFi.

Conclusion: Conduction defects were rare in this group of axSpA patients. Only $3 \%$ had prolonged AV conduction, which is no higher than general population estimates [1]. The prognostic value of these conduction defects and QT dispersion requires further study.

References:

[1] van der Ende et al. Population-based values and abnormalities of the electrocardiogram in the general Dutch population: The LifeLines Cohort Study. Clin Cardiol. 2017; 40(10): 865-872

Table 1. ECG measurements in 163 axSpA patients.

\begin{tabular}{lccc}
\hline & Mean (SD) & & $\mathbf{n}(\%)$ \\
\hline PR, ms & $149(24)$ & PR>200ms & $5(3 \%)$ \\
QRS, ms & $91(15)$ & QRS $>100 \mathrm{~ms}$ & $25(16 \%)$ \\
QTc, ms & $385(32)$ & QTc prolonged & $6(4 \%)$ \\
QT dispersion, ms & $43(21)$ & QT dispersion $>50 \mathrm{~ms}$ & $41(26 \%)$ \\
\hline
\end{tabular}

Disclosure of Interests: None declared

DOI: 10.1136/annrheumdis-2020-eular.3179

\section{AB0730 ASSOCIATION OF SPONDYLOARTHRITIS AND FAMILIAL MEDITERRANEAN FEVER AND IMPACT ON DISEASE PHENOTYPE: A SYSTEMATIC REVIEW OF THE LITERATURE}

N. Ziade ${ }^{1}$, A. Nassar' ${ }^{1}{ }^{1}$ Saint-Joseph University, Beirut, Lebanon

Background: Spondyloarthritis (SpA) and Familial Meditaerranean fever (FMF) may co-exist in certain populations, and have some overlapping manifestations (oligo-arthritis, hip involvement). Their association may impact disease phenotype and may affect disease management.

Objectives: To evaluate the association of $\mathrm{SpA}$ and FMF and its impact on disease phenotype and management.

Methods: A systematic literature search was conducted with the keywords spondyloarthritis and familial mediterranean fever from Janurary 1990 to January 2020 in PubMed and using manual cross-reference methods.
Results: The search retrieved 74 articles, out of which 37 articles were relevant to the study question; most of the articles were case reports, with some large cohort studies of FMF and SpA (Flowchart in Figure 1).

In large FMF cohorts, the prevalence of SpA was higher compared to the general population $(7.5-13 \%$, OR around 10$)$. M694V was a risk factor for SpA. These FMF-SpA patients were older at diagnosis, had lower fever attacks, and higher disease duration, inflammatory back pain, chronic arthritis, enthesopathy, persistent inflammation and higher resistance to Colchicine. In case series, they were responsive to anti-TNF therapy.

In large SpA cohorts, MEFV mutation, particularly M694V, was found in $15-35 \%$ (even without associated FMF). In most cohorts, MEFV mutation carriers didn't have any distinct disease phenotype, except for some reports of higher ESR more hip involvement, higher BASFI and higher BASDAI. Genome-wide association studies and case reports suggest an implication for IL-1 and thus a role for Anakinra therapy in these patients.

Conclusion: In FMF or SpA patients with resistance to conventional therapy, the evaluation of disease association should be performed as it may have significant impact on disease management.

References:

[1] Li et al, Plos Genetics 2019. Watad et al, Frontiers Immunol 2019. Atas et al, Rheumatol Int 2019. Cherqaoui et al, JBS 2017. Zhong et al. Plos One 2017 Ornek et al, Arch Rheumatol 2016. Cinar et al, Rheumatol Int 2008. Durmur et al, JBS 2007

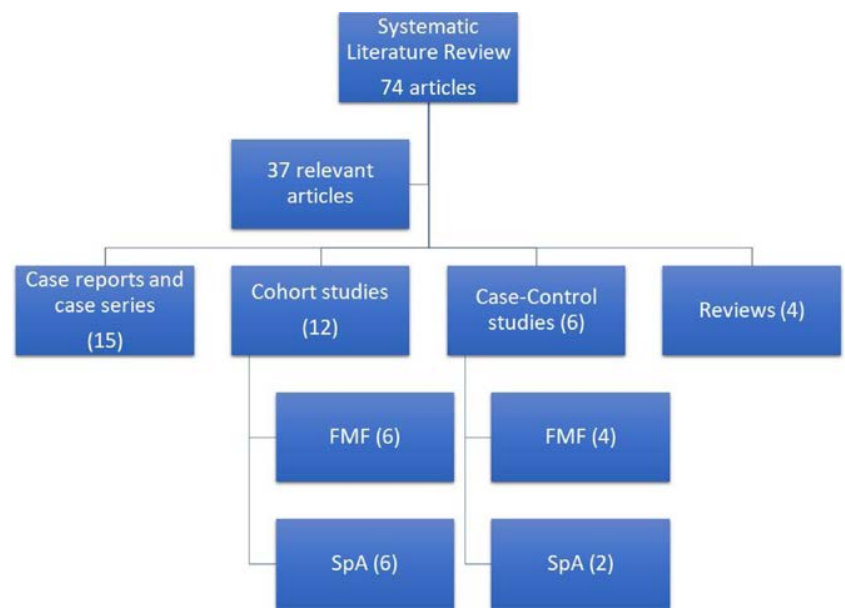

Figure 1. Flowchart of the systematic literature search (Spondyloarthritis, Familial Mediterranean Fever; January 1990-2020).

Disclosure of Interests: Nelly Ziade Speakers bureau: Abbvie, Janssen, Lilly, Novartis, Pfizer, Roche, Sanofi, Aref Nassar: None declared DOI: 10.1136/annrheumdis-2020-eular.6009

\section{Psoriatic arthritis}

\begin{tabular}{|l|l}
\hline AB0731 & IMPACT OF FIBROMYALGIA ON DISEASE ACTIVITY \\
INDICES, HEALTH RELATED QUALITY OF LIFE AND \\
FATIGUE IN PATIENTS WITH PSORIATIC ARTHRITIS
\end{tabular}

N. Elsawy ${ }^{1}$, M. Helal ${ }^{1}$, H. Ashraf Abd Elhamid ${ }^{1}$, Y. Abdel-Fattah ${ }^{1} .{ }^{1}$ Faculty of Medicine Alexandria University, Physical Medicine, Rheumatology and Rehabilition, Alexandria, Egypt

Background: Psoriatic arthritis (PsA), causes inflammation in joints and enthesis, emotional instability and poor quality of life (QOL). ${ }^{1}$ Fibromyalgia (FM) may coexist with PsA, complicating its diagnosis and management. ${ }^{2}$ The effect of FM on the QOL and fatigue in PsA patients has not been vastly studied. ${ }^{3}$

Objectives: Assess the effect of FM on PsA patients' disease activity indices, QOL and fatigue.

Methods: This study included Group I: 37 PsA only patients $(61.7 \%), 48.38 \pm 11.69$ years and group II: 23 FM-PsA patients (38.3\%), $50.78 \pm 11.8$ years, according to classification criteria for PsA and 2016 Revisions to 2010/2011 FM diagnostic criteria Psoriasis area severity index (PASI), disease activity in PSA (DAPSA), composite PSA activity index (CPDAI), PsA QOL and multidimensional assessment of fatigue (MAF) were assess in both groups. The severity and impact of FM was assessed in group II. Results: Patients with FM-PsA had a statistically higher PsA disease activity in subjective measures only but not in objective measures. Table 1 